\title{
In Vitro Chloroquine Resistance in Plasmodium falciparum Isolates from Tertiary Care Hospital
}

\author{
Fatima Shujatullah, Haris M. Khan, Abida Khatoon, Parvez A. Khan, and Mohammad Ashfaq \\ Department of Microbiology, Jawaharlal Nehru Medical College, Aligarh Muslim University, Aligarh 202001, India \\ Correspondence should be addressed to Fatima Shujatullah, sfatima777@gmail.com
}

Received 27 May 2012; Revised 3 August 2012; Accepted 29 August 2012

Academic Editor: Neena Valecha

Copyright (๑) 2012 Fatima Shujatullah et al. This is an open access article distributed under the Creative Commons Attribution License, which permits unrestricted use, distribution, and reproduction in any medium, provided the original work is properly cited.

\begin{abstract}
Chloroquine (CQ) has been the mainstay of treatment of malaria for decades. This cost-effective and safe drug has become ineffective for treatment of falciparum malaria in many parts of the world due to development of resistance by the parasite. In addition CQ is not gametocytocidal for P. falciparum and thus cannot block transmission. The extent of problem of chloroquine resistance in $P$. falciparum is increasing every year. The study was done in period of 2 years. A total of 5653 specimens were examined for malarial infection by employing different diagnostic modalities. Four hundred and thirty-five were found to be positive for $P$. falciparum by using different diagnostic techniques. All positive specimens were cultured on RPMI 1640 medium; only 108 were found to be culture positive. Sensitivity of isolates to chloroquine was done using Mark III WHO sensitivity plates. The prevalence of malaria infection was found $9.54 \%$ in 2010 . There were schizont formation at 8 pmol/liter or more of chloroquine concentration in 26 isolates. The emergence of chloroquine (CQ) resistance pattern in Aligarh isolates increases. Antimalarial agents should be used with caution; monotherapies should be avoided.
\end{abstract}

\section{Introduction}

Chloroquine has for decades been the primary chemotherapeutic means of malaria treatment and control. This safe and inexpensive 4-aminoquinoline compound accumulates inside the digestive vacuole of the infected red blood cell. Chloroquine resistance (CQR) was first reported in Southeast Asia and South America and has now spread to the vast majority of malaria-endemic countries [1]. Antimalarial drug resistance is a major public health problem which hinders the control of malaria. In India resistance of Plasmodium falciparum to chloroquine was first reported in the year 1973 from Diphu of the Karbi Anglong district in Assam state.

Various in vitro sensitivity test systems have been developed and applied to sensitivity monitoring of $P$. falciparum in endemic areas. The most commonly used methods are in vitro tests based on the measurement of the effect of drugs on the growth and development of malaria parasites, that is, schizont maturation or growth inhibition $[2,3]$, incorporation of radiolabeled precursors [4], enzymatic activity of parasite lactate dehydrogenase (pLDH) [5], or histidine-rich protein II (HRP II) [6]. The in vitro sensitivity test based on the standard micro-technique recommended by the World Health Organization [2] using the schizont maturation inhibition test has been applied successfully in most of the highly multi-drug-resistant areas worldwide. The study was done to determine the prevalence of $P$. falciparum infection in Aligarh, a north Indian district and to test the chloroquine sensitivity using Mark III WHO sensitivity plates.

\section{Material and Method}

2.1. Place of Study and Study Cohort. The study was conducted in Aligarh, a district of north India, where there is high prevalence of $P$. falciparum species. The study was conducted from February 2010 to December 2011, but majority of cases $(>90 \%)$ were detected in months of July to October in both years. A total of 5653 patients, who presented with fever, chills, rigors, or any pyrexia of unknown origin were screened for malarial infection by using different diagnostic tests 
like peripheral blood smear examination and quantitative buffy coat assay and by various antigen detection assays. The blood was cultured from every positive case of $P$. falciparum. Parasite density was not taken into account. The drug sensitivity was done against chloroquine as it is the most commonly used antimalarial agent in out-patient departments for uncomplicated malarial infection. It is also prescribed empirically even before the laboratory diagnosis is confirmed. We also want to study chloroquine sensitivity because of its improper prescription and dosing in fever of unknown origin in this area by unqualified medical practitioners.

2.2. Specimen Collection. $5 \mathrm{~mL}$ of blood was collected by venipuncture taking all sterile precaution in heparinized tube. Both thick and thin smears were examined by staining with Giemsa stained using standard protocols. Parasitemia was determined by counting 200 WBCs on thick films and expressed as parasites per microliter of blood. A total of 200 oil emergence fields were examined before the smear is labeled negative or positive.

\subsection{In Vitro Cultivation of Plasmodium falciparum Isolates} and Drug Sensitivity Test. The in vitro cultivation of $P$. falciparum isolates followed a modification of the standard culture techniques $[7,8]$. The culture medium consisted of RPMI 1640 (Sigma Aldrich), $2 \mathrm{~g}$ glucose, and $40 \mu \mathrm{g} / \mathrm{mL}$ gentamycin sulphate with supplemented $10 \% \mathrm{AB}+$ serum. Culture medium was sterilised by filtration through a Millipore filter of $0.22 \mu \mathrm{m}$ porosity and $\mathrm{pH}$ was adjusted to 7.4 by the addition of $4.2 \mathrm{~mL}$ of sterile $5 \%$ sodium bicarbonate.

The drug sensitivity (based on the M-III methods) can be determined in vitro in $P$. falciparum culture by using standard 96-well microtitre plates (WHO plates), predosed of the test drug. The test plates were predosed with ascending concentrations of chloroquine A-H $0,1,2,4,8,16,32$, and $64 \mathrm{pmol}$. Blood medium mixture (BMM) was prepared by gently shaking the tube to mix the blood and medium. Preculture thick and thin films were prepared. All the wells of appropriate column were dosed with $50 \mu \mathrm{L}$ of the blood medium mixture $(1: 9)$.

The plate was put in a candle jar and placed in the incubator set at $37.5^{\circ} \mathrm{C}$ for $24-30 \mathrm{hrs}$, depending upon development stage of the parasite. After 24-hour incubation, a thin smear was prepared from the control well to see the mature schizonts, and if more than $10 \%$ schizonts were seen, it was considered to be valid; thick smears were prepared from each well by discarding the excess media with a micropipette.

2.4. Determination of In Vitro Effective Concentration (EC) Values of the Chloroquine. The mean number of schizonts counts per well was fed directly into nonlinear regression software, $\mathrm{H}$ N-Non-Lin V1.1, specific for malaria in vitro drug sensitivity test. Individual dose response curves were generated and their EC 50, EC 90, and EC 99 values determined.
TABLe 1: Clinical features in 435 patients of $P$. falciparum infection.

\begin{tabular}{lcc}
\hline S. no. & Clinical features & No. of patients \\
\hline$(1)$ & Fever & $435(100 \%)$ \\
$(2)$ & Chills & $372(85.51 \%)$ \\
$(3)$ & Rigors & $310(71.26 \%)$ \\
$(4)$ & Convulsions & $156(35.86 \%)$ \\
$(5)$ & Neck rigidity & $103(23.67 \%)$ \\
$(6)$ & Other signs of meningeal irritation & $79(18.16 \%)$ \\
$(7)$ & Yellowish discoloration of sclera & $45(10.34 \%)$ \\
$(8)$ & Renal complaints & $39(8.96 \%)$ \\
$(9)$ & Altered consciousness & $325(60.74 \%)$ \\
\hline
\end{tabular}

2.5. Data Analysis. The geometric means and 95\% confidence intervals (CIs) of EC values were estimated in SPSS (SPSS Inc., USA).

2.6. Observations. Out of 5653 patients who presented with pyrexia of unknown origin or pyrexia associated with symptoms suggestive of malarial infection, only 435 patients had $P$. falciparum infection. Fever was present in all patients $(100 \%)$; chills (85.51), rigors (71.26\%), and altered level of consciousness $(60.74 \%)$ were the common features with which $P$. falciparum positive cases presented (Table 1). Four hundred and thirty-five $P$. falciparum-positive blood samples were cultured; 108 cultures were positive; from all these isolates when subjected to chloroquine sensitivity, 26 isolates were found to be resistant to chloroquine, that is, schizont maturation at 8 pmols or more. Isolates showed schizont maturation up to 32 pmols or more (Figure 1). The mean effective concentrations of chloroquine EC $50=$ $6.07 \mathrm{~nm} /$ liter, EC $90=33.33 \mathrm{~nm} /$ liter, EC $95=43.44 \mathrm{~nm} / \mathrm{liter}$, and EC $99=47.40 \mathrm{~nm} /$ liter, which shows decreased sensitivity of $P$. falciparum isolates to chloroquine (Table 2).

\section{Discussion}

The major obstacle in malaria treatment and control is impeded by the drug-resistant parasites, particularly Plasmodium falciparum, which have disseminated and enhanced mortality [9]. Chloroquine drug pressure remains high in India as it has been used since decades as first-line drug in malaria therapy both as self-treatment at home and in health-care facilities [10] Shah et al. [11] analyzed 337 studies and investigated chloroquine efficacy in 17189 patients. The number of studies and proportion of failures varied between regions and in states within a region. The median proportion of chloroquine failure was $35.1 \%$ (IQR 13.0-58.2) in studies with a 28-day followup. Studies with 7-day followup, which largely detected early treatment failures, were phased out from 2000 and the last 7-day study was done in 2003. The proportion of failures detected is higher in 28-day followup studies than in 7-day follow-up studies done in the same areas, because late treatment failures are detected in 28-day follow-up studies. Studies done between 1978 and 2007 show an increasing proportion of failures to chloroquine over time. 
TABLE 2: Geometric mean EC 50, EC 90, and EC 99, 95\% confidence interval (CI) of chloroquine against Plasmodium falciparum isolates.

\begin{tabular}{lcc}
\hline Chloroquine & \multicolumn{2}{c}{ Geometric mean EC 50, (95\% CI) nanomolar (nm/liter) } \\
& Sensitive isolates & $6.07(02.74-13.42)$ \\
EC 50 & $1.30(1.99-1.0)$ & $33.33(16.94-61.09)$ \\
EC 90 & $3.94(5.53-1.88)$ & $43.44(19.15-65.78)$ \\
EC 95 & $5.56(7.22-2.48)$ & $47.40(21.39-66.98)$ \\
EC 99 & $6.17(1.0-0.92)$ & \\
\hline
\end{tabular}

EC: effective concentration.

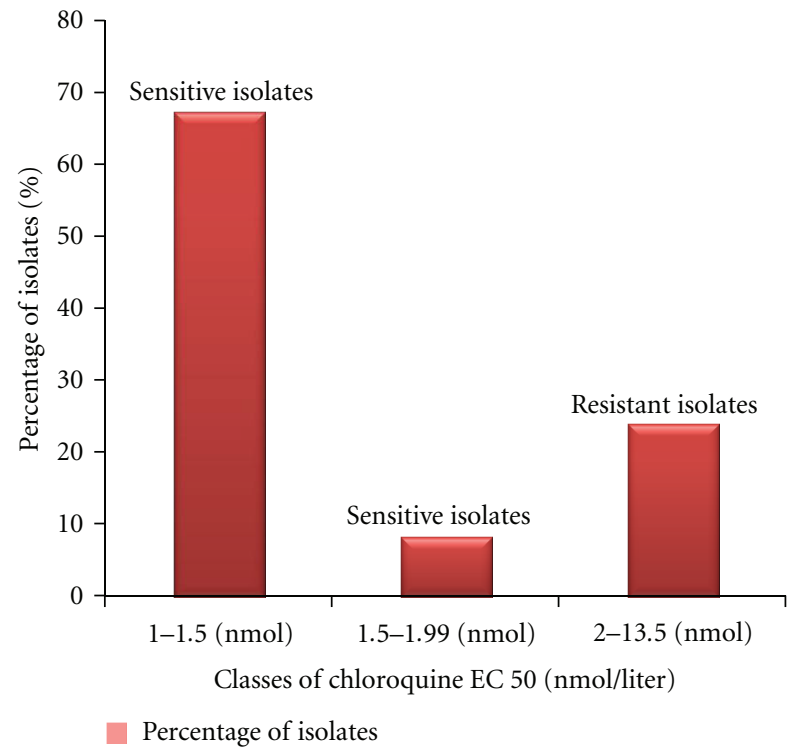

Figure 1: Relative EC 50 (nmol/liter). Distribution pattern of chloroquine among $P$. falciparum isolates.

When routine monitoring of drug resistance began in 19781979, two of 17 studies exceeded the 10\% threshold used in India to switch an area to the second-line treatment. In 2006-2007, the proportion of chloroquine studies exceeding $10 \%$ treatment failure increased to 35 of 40 studies. Drugefficacy studies of chloroquine, with at least 30 patients in any follow-up period, have exceeded $10 \%$ treatment failure in 115 districts. These districts represent 20 of 28 states and two of five union territories. The remaining states and territories have low incidence of $P$. falciparum, or have not done any trial of antimalarial drug resistance [11]. Culture was positive in 108 patients with confirmed malaria diagnosis. Only $31 \%$ cases have given adequate culture results due to low parasitemia, previous history of inadequate treatment by quacks. This is a tertiary care hospital where most of the patients come after getting treatment from one or more sources.

Most of the studies done in India mainly determined the in vivo resistance patterns of chloroquine [12]. There is paucity of data from India regarding in vitro drug resistance pattern in India. In our study when chloroquine was tested against $P$. falciparum isolates, high level of resistance was noticed by in vitro Mark III sensitivity assay $53.76 \%$ of isolates resistant to 8 pmols of chloroquine which is the WHO cut-off value for sensitivity. The level of resistance in this part of India is so high that $10.75 \%$ strains were resistant to even 32 pmols of drug. One of the major objectives of the study is to determine effective concentration values (EC values) of chloroquine. This result is needed to estimate the effective therapeutic levels in human body. Our results showed that mean EC5O value of sensitive strains was 1.30 and for resistant 6.07 (nmol/liter), EC 90, EC 95, and EC 99 were found to be $3.94 \mathrm{~nm} /$ liter and $33.33 \mathrm{~nm} /$ liter, $5.56 \mathrm{~nm} /$ liter and $43.44 \mathrm{~nm} /$ liter, $6.17 \mathrm{~nm} /$ liter, and $47.40 \mathrm{~nm} /$ liter for sensitive and resistant isolates, respectively. These results indicate high level of resistance of $P$. falciparum to chloroquine among the isolates tested, and this is confirmed by high EC values which are excessively higher than effective therapeutic concentration. These values are significantly higher than those reported from India and other countries [13, 14]. This high-level resistance to chloroquine in our patients may be due to previous exposure to improper therapeutic regimes, over-the-counter availability of drug, and high drug pressure by improper prescribing habits of private practitioners. So this high level of resistance should be monitored regularly to prevent further spread of resistant strains. The use of combination therapy should be advocated to prevent the drug resistance in malaria-endemic areas.

\section{References}

[1] R. G. Ridley, "Medical need, scientific opportunity and the drive for antimalarial drugs," Nature, vol. 415, no. 6872, pp. 686-693, 2002.

[2] K. H. Rieckmann, G. H. Campbell, L. J. Sax, and J. E. Mrema, "Drug sensitivity of Plasmodium falciparum. An in-vitro microtechnique.," Lancet, vol. 1, no. 8054, pp. 22-23, 1978.

[3] World Health Organization, In Vitro Microtest (MARK II) for the Assessment of the Response of Plasmodium Falciparum to Chloroquine, Mefloquine, Quinine, Sulfadoxine/Pyrimentamine and Amodiaquine, World Health Organization, Geneva, Switzerland, 1990.

[4] R. E. Desjardins, C. J. Canfield, J. D. Haynes, and J. D. Chulay, "Quantitative assessment of antimalarial activity in vitro by a semiautomated microdilution technique," Antimicrobial Agents and Chemotherapy, vol. 16, no. 6, pp. 710-718, 1979.

[5] M. T. Makler and D. J. Hinrichs, "Measurement of the lactate dehydrogenase activity of Plasmodium falciparum as an assessment of parasitemia," American Journal of Tropical Medicine and Hygiene, vol. 48, no. 2, pp. 205-210, 1993.

[6] H. Noedl, W. H. Wernsdorfer, R. S. Miller, and C. Wongsrichanalai, "Histidine-rich protein II: a novel approach to 
malaria drug sensitivity testing," Antimicrobial Agents and Chemotherapy, vol. 46, no. 6, pp. 1658-1664, 2002.

[7] W. Trager and J. B. Jensen, "Human malaria parasites in continuous culture," Science, vol. 193, no. 4254, pp. 673-675, 1976.

[8] J. D. Haynes, C. L. Diggs, F. A. Hines, and R. E. Desjardins, "Culture of human malaria parasites Plasmodium falciparum," Nature, vol. 263, pp. 767-769, 1976.

[9] P. J. Guerin, P. Olliaro, F. Nosten et al., "Malaria: Current status of control, diagnosis, treatment, and a proposed agenda for research and development," Lancet Infectious Diseases, vol. 2, no. 9, pp. 564-573, 2002.

[10] A. Ahmed, D. Bararia, S. Vinayak et al., "Plasmodium falciparum, Hyde JE. Drug-resistant malaria," Trends Parasitol, vol. 21, pp. 494-483, 2005.

[11] N. K. Shah, G. P. S. Dhillon, A. P. Dash, U. Arora, S. R. Meshnick, and N. Valecha, "Antimalarial drug resistance of Plasmodium falciparum in India: changes over time and space," The Lancet Infectious Diseases, vol. 11, no. 1, pp. 57-64, 2011.

[12] R. N. Prasad, H. Prasad, K. J. Virk, and V. P. Sharma, "Application of a simplified in-vivo test system for determining chloroquine resistance in Plasmodium falciparum," Bulletin of the World Health Organization, vol. 68, no. 6, pp. 755-758, 1990.

[13] V. K. Dua, P. K. Kar, S. Kumar, and V. P. Sharma, "In-vivo and in-vitro sensitivity of Plasmodium falciparum to chloroquine at Indian Oil Corporation, Mathura (U.P.)," Indian Journal of Malariology, vol. 30, no. 1, pp. 29-35, 1993.

[14] H. Al-Shamahy, A. H. Al-Harazy, N. S. Harmal, and A. M. AlKabsi, "The prevalence and degree of resistance of Plasmodium falciparum to first-line antimalarial drugs: an in vitro study from a malaria endemic region in Yemen," Annals of Saudi Medicine, vol. 27, no. 6, pp. 432-436, 2007. 


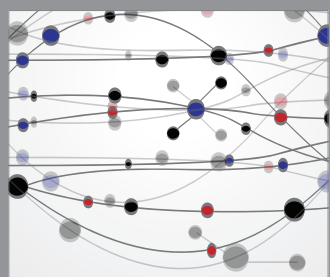

The Scientific World Journal
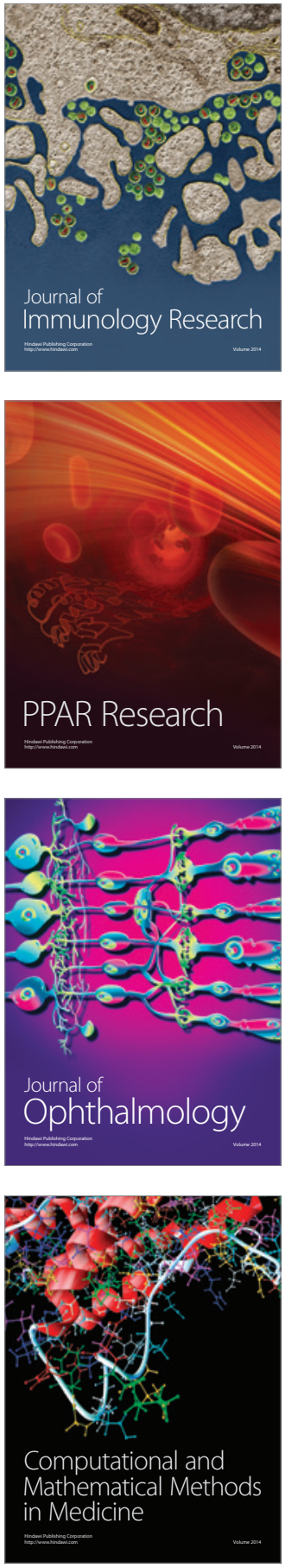

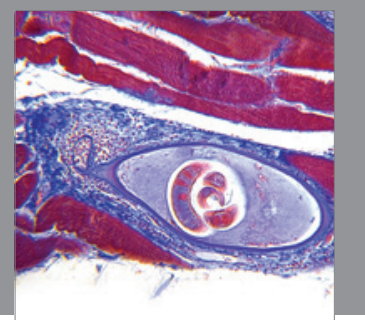

Gastroenterology

Research and Practice
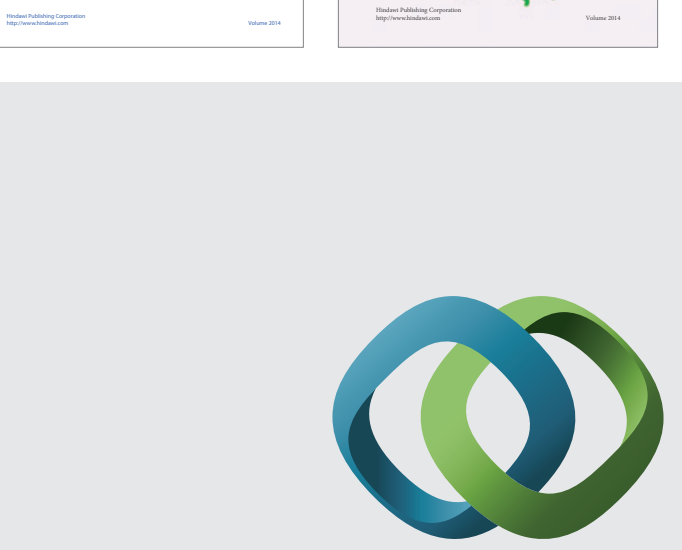

\section{Hindawi}

Submit your manuscripts at

http://www.hindawi.com
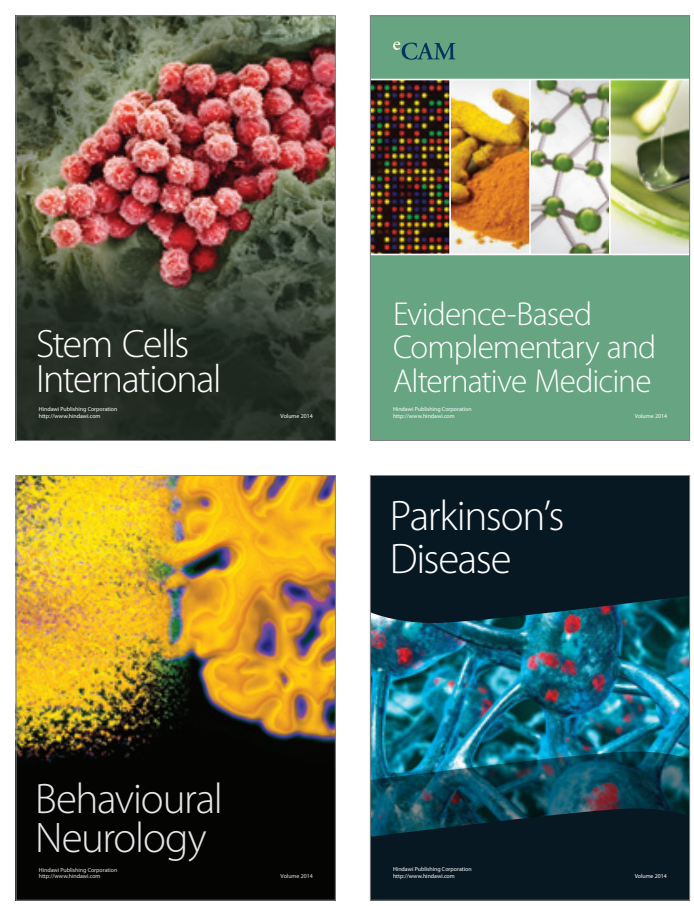

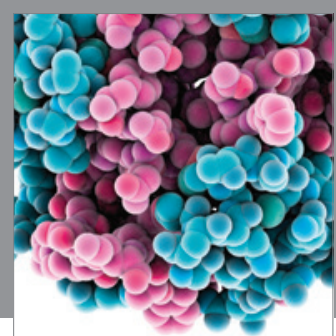

Journal of
Diabetes Research

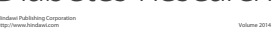

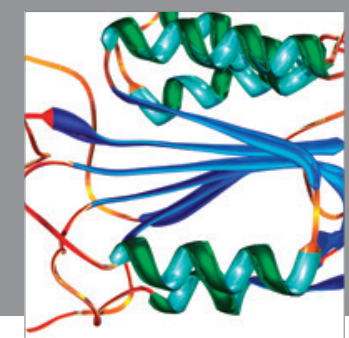

Disease Markers
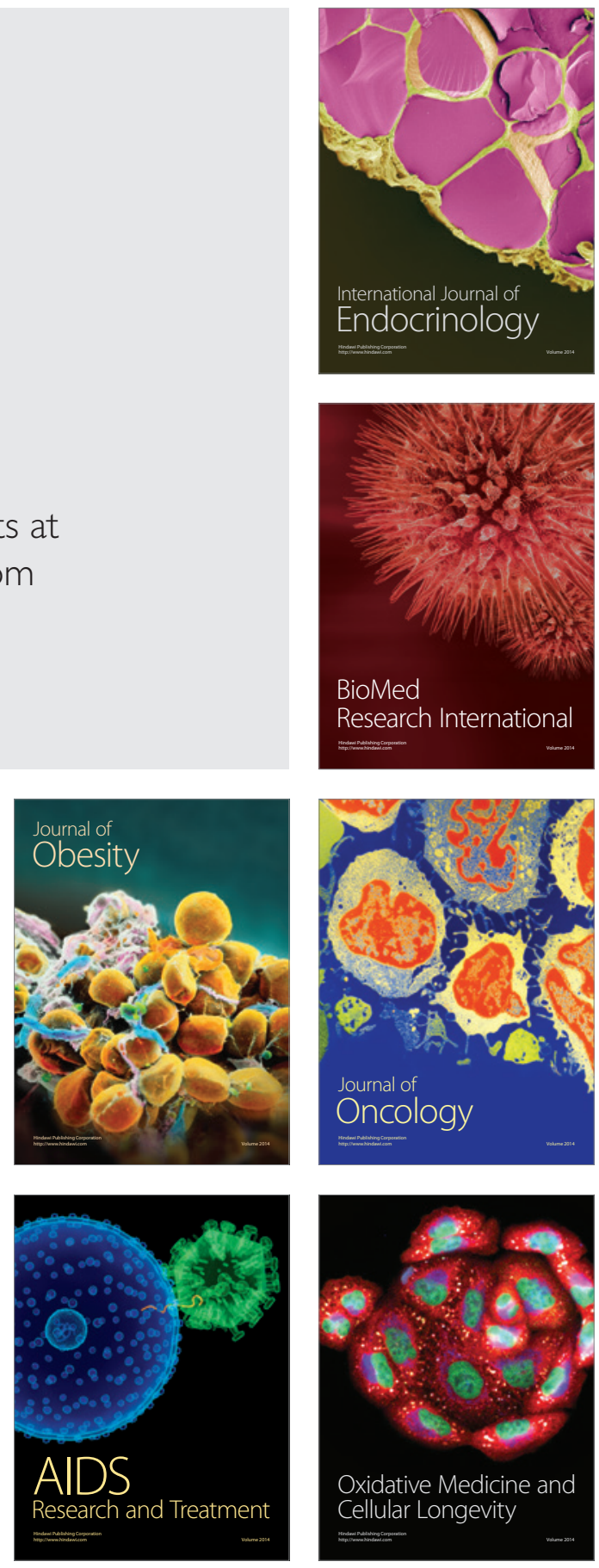